\title{
Grado de conocimiento y aplicación de las nuevas tecnologías de la información y de la comunicación en la docencia de la electrocardiografía por los médicos de la provincia de Toledo
}

\author{
Luis Rodríguez-Padial, María Luz Cacheiro-González
}

Introducción. Las nuevas tecnologías de la información y de la comunicación (TIC) han contribuido al desarrollo científico y social y desempeñan un papel esencial en la docencia y, en especial, en la medicina. La electrocardiografía es una técnica útil en la práctica clínica, de forma que muchos médicos deben estudiarla. Aunque se han utilizado las TIC, su grado de implantación está limitado, por lo que se pretende conocer el grado de conocimiento y aplicación de las TIC por los médicos de la provincia de Toledo en el aprendizaje de la electrocardiografía.

Sujetos y métodos. Se ha enviado un cuestionario por Internet (Google Docs) a todos los médicos que tienen correo electrónico con cuestiones sobre el uso y la opinión de las TIC. Se realiza un análisis estadístico y se pretende conocer el grado de conocimiento y aplicación de las TIC.

Resultados. Se han recogido 199 cuestionarios. La edad media es de 46,6 \pm 9,7 años (rango: 25-67 años), siendo el 56\% varones. El $90 \%$ de los médicos utiliza los ordenadores, pero usan otros medios con una frecuencia mucho menor. Un $52 \%$ utilizan teléfonos inteligentes. El 74\% ha realizado algún aprendizaje de electrocardiografía, teniendo la mayoría un nivel básico. Tienen mayor experiencia en métodos tradicionales e individuales que en las TIC. Creen que las TIC pueden ser útiles, aunque valoran más las visitas a la web y las aplicaciones portátiles que los edublogs.

Conclusiones. En este colectivo existe interés por la electrocardiografía y se observa una importante deficiencia en el uso de las TIC.

Palabras clave. Docencia. Electrocardiografía. Técnicas de información y comunicación.

Degree of knowledge and implementation of new information technology to learn electrocardiography by doctors from the province of Toledo

Introduction. New advances in information technology (IT) have contributed to the social and scientific development of modern society, and have played a significant role in teaching, especially in medicine. Electrocardiography is a useful diagnostic technique in medicine and many doctors must study it in clinical practice. IT can be very helpful when learning electrocardiography but its usefulness is hampered by doctors' limited knowledge of IT. The aim of this study is to understand the degree of knowledge and use of IT when doctors from the province of Toledo learn about electrocardiography.

Subjects and methods. A questionnaire (Google Docs) was sent by e-mail from the Medical Council to all doctors that use e-mail in their relationship with the Council. A statistical analysis was performed to study the degree of knowledge and use of IT.

Results. A total of 199 questionnaires were analysed. The average age was $46.6 \pm 9.7$ years-old (range: $25-67$ years-old), $56 \%$ of the participants were men. Most doctors (90\%) use computers, but employ other forms of IT less frequently. Smartphones are used by $52 \%$ of them. Most (74\%) studied electrocardiography, having a basic level of understanding. More experience is shown in classic and individual methods than in IT. While most of the doctors think that IT can be useful, they value websites and smartphone applications more than they value educational blogs.

Conclusions. This group of doctors show interest in electrocardiography but demonstrate a significant deficiency in the use of IT. Key words. Electrocardiography. Information technologies. Teaching.
Departamento de Didáctica, Organización Escolar y Didácticas Especiales; Facultad de Educación UNED (M.L. Cacheiro-González). Servicio de Cardiología; Complejo Hospitalario de Toledo (L. RodríguezPadial). Toledo, España.

Correspondencia:

Dr. Luis Rodríguez Padial. Servicio de Cardiología. Complejo Hospitalario de Toledo. Avda. Barber, 30. E-45004 Toledo

E-mail:

Irpadial@gmail.com

Agradecimientos: Al Ilustre Colegio Oficial de Médicos de Toledo, por su colaboración en el envío de las encuestas, y al Dr. Francisco Martín Rodríguez, por su apoyo en el análisis estadístico.

Nota:

El apéndice citado en este artículo puede consultarse en su versión electrónica: www. fundacioneducacionmedica.org.

Conflicto de intereses: No declarado.

Competing interests: None declared.

(c) 2014 FEM 


\section{Introducción}

Las tecnologías de la información y la comunicación (TIC) están contribuyendo de manera decisiva a cambiar la sociedad y la forma en la que nos comunicamos. Han tenido un gran impacto en la educación de diversas disciplinas y a distintos niveles, de manera que están produciendo una transformación global de la educación [1].

La medicina es una ciencia en constante transformación gracias a los continuos avances científicos. Las TIC puedan tener un papel de gran relevancia en diversos aspectos de la medicina, desde la promoción de la salud hasta el seguimiento de pacientes crónicos, donde pueden contribuir a mejorar la eficiencia de la asistencia sanitaria [2,3]. Pero el impacto que las TIC han realizado en la medicina no se centra exclusivamente en el campo de la atención, sino también, y de forma muy notable, en el de la formación médica, en la que se están produciendo importantes transformaciones al orientarla a la solución de problemas y al desarrollo de capacidades $[4,5]$. En este terreno, la expansión de las TIC se ve limitada por el conocimiento y uso que los médicos tienen de ellas, que suele ser limitado [6-10].

La electrocardiografía es una técnica diagnóstica que debe ser estudiada por la mayoría de los médicos para su práctica clínica, a pesar de lo cual se ha demostrado una formación habitualmente deficiente [11-16]. Se han realizado pocos estudios sobre el método más adecuado para la docencia de la electrocardiografía, habiendo sido los procedimientos habituales (autoaprendizaje con libros, cursos presenciales, etc.) los métodos utilizados con mayor frecuencia, aunque otros, incluida la danza [17], han demostrado ser eficaces [18-20].

Recientemente se han comunicado experiencias de enseñanza de la electrocardiografía mediante la utilización de las TIC $[21,22]$. En la actualidad, existen numerosas páginas web destinadas a la difusión y docencia de la electrocardiografía, así como algún blog educativo (edublog). No obstante, en nuestro medio existe escasa información sobre el uso que los médicos hacen de las TIC en el aprendizaje y, en especial, en el de la electrocardiografía.

\section{Objetivo}

El objetivo de este trabajo ha sido conocer el grado de conocimiento que los médicos de la provincia de Toledo tienen sobre las nuevas TIC aplicadas a la docencia/aprendizaje de la electrocardiografía.

\section{Sujetos y métodos}

\section{Muestra}

Se ha remitido por correo electrónico una encuesta a todos los médicos inscritos en el Colegio Oficial de Médicos de Toledo que utilizan el correo electrónico para sus relaciones con el colegio, 2.154 de un total de 2.478 médicos colegiados.

\section{Encuesta}

A través de Google Docs [23] se ha realizado una encuesta de 56 preguntas, 20 de las cuales se dirigen a la caracterización de la persona encuestada (Apéndice). El cuestionario se ha realizado con preguntas cerradas de escalas de medición de actitudes y respuestas, utilizando la escala de valoración de Likert con cinco respuestas e incluyendo variables de entrada (10 cuestiones sobre datos demográficos), de proceso (12 cuestiones sobre grado de uso de las TIC en la vida diaria) y de producto (13 cuestiones sobre experiencia en estudio de la electrocardiografía y en el uso de las TIC para ello). Se añadieron otras 11 cuestiones sobre la opinión que los encuestados tenían sobre el uso potencial de las TIC en el aprendizaje de la electrocardiografía. En las preguntas referentes a determinadas técnicas, de menor difusión y conocimiento, se acompañaron explicaciones breves sobre ellas para facilitar una selección basada en un mayor conocimiento. Las respuestas se clasifican como: 1 , totalmente en desacuerdo (o desconocido); 2 , en desacuerdo; 3 , indiferente; 4 , de acuerdo, y 5, totalmente de acuerdo (o conocido) [24] Se ha preguntado por las TIC consideradas por Cabero-Almenara y Román-Graván [25].

Para evaluar la validez del cuestionario se realizó una evaluación por pares y un análisis de fiabilidad según el método de consistencia interna $\alpha$ de Cronbach. Se remitió a un grupo de expertos en metodología de la investigación y docencia (D.J. Gallego, M. Carretero) y en electrocardiografía (D. Marzal), al objeto de obtener sugerencias sobre el mismo. Se valoró la idoneidad de las preguntas, la fluidez del cuestionario y su capacidad de discriminación, entre otros aspectos del diseño. Las correspondientes sugerencias y comentarios se introdujeron en el cuestionario definitivo. De nuevo, antes de remitir el cuestionario al total de los médicos encuestados se realizó un estudio piloto, enviando el cuestionario a un grupo aleatorio de 50 médicos seleccionados entre el grupo total de estudio, al objeto de realizar el análisis de consistencia interna según el $\alpha$ de Cronbach utilizando el programa estadístico SPSS. 
De estos registros se recogieron 44 respuestas, que fueron las incluidas en al análisis de fiabilidad. Con estos cuestionarios se obtuvo una $\alpha$ de Cronbach de 0,89 , que se consideró adecuada al ser mayor de 0,7 , el límite considerado habitualmente como indicativo de adecuada fiabilidad. El $\alpha$ de Cronbach obtenido en cada uno de los módulos fue superior a 0,75 (osciló entre 0,75 y 0,90). Tras comprobar la consistencia interna del cuestionario se procedió a realizar su envío a todos los médicos colegiados.

\section{Análisis estadístico}

Los datos cualitativos se han expresado como proporción, y los cuantitativos, como media \pm desviación estándar o mediana más rango. Se han analizado las diferencias entre proporciones mediante la chi al cuadrado. De igual forma, se han estudiado las diferencias entre los médicos menores y mayores de 40 años en el uso que hacen de las TIC.

Se ha analizado la diferencia y la correlación (coeficiente de correlación de Pearson) entre la puntuación obtenida entre el grado de conocimiento y la opinión que los médicos tienen sobre las distintas TIC consideradas.

Para el análisis estadístico se ha usado el programa SPSS v. 15.0. En las comparaciones de dos colas se ha considerado significativo un valor $p<0,05$.

\section{Resultados}

\section{Datos demográficos}

Se han completado 199 encuestas, de las cuales el $56 \%$ corresponden a varones. La edad media de los encuestados ha sido de 46,6 \pm 9,7 años (rango: 25-67 años). De éstos, 54 tienen una edad igual o inferior a 40 años. El 5,5\% de los encuestados son de nacionalidad no española (iberoamericanos). El año de finalización de la licenciatura en medicina oscila entre 1969 y 2011 . El 57\% de ellos trabaja en el medio urbano, perteneciendo el 83\% al Área Sanitaria de Toledo, y el resto (16\%), al Área Sanitaria de Talavera de la Reina. El 7\% de los encuestados trabaja, además, en otra provincia española. El 10\% de los que han respondido la pregunta son médicos residentes.

En cuanto a la especialidad médica, el 55\% corresponde a atención primaria, el $12 \%$ a cardiología y el 33\% restante tiene otra especialidad médica o quirúrgica. Ninguno de los encuestados estaba adscrito a la universidad. El 74\% de los encuestados ha realizado algún tipo de aprendizaje de la electrocardiografía, mientras que el $26 \%$ indica que nunca ha intentado aprender esta técnica diagnóstica. En cuanto al grado de conocimiento de la técnica, el 13\% tiene un nivel avanzado; el 38\%, un nivel intermedio; el $49 \%$, un nivel básico, y el $2 \%$ reconoce no tener conocimiento alguno sobre esta técnica diagnóstica.

\section{Experiencia en el uso de las TIC en tareas personales}

De los encuestados, el 90\% utiliza los ordenadores (para algo más que para correo electrónico y trabajo); el 60\%, los videoproyectores; el 61\%. los CD interactivos; el 63\%, los equipos multimedia, y el 91\%, Internet y el correo electrónico. Por el contrario, la webcam es utilizada por sólo un $38 \%$, y el equipo multimedia, por un $27 \%$. El $52 \%$ de los encuestados usa los teléfonos inteligentes (iPhone y similares), y el 36\%, las tabletas electrónicas (iPad y similares).

\section{Importancia de la enseñanza-aprendizaje de la electrocardiografía}

Con respecto a la importancia dada a la enseñanza de la electrocardiografía en la universidad, el $23 \%$ es indiferente (puntuación de 3), mientras que el $49 \%$ piensa que se le da poca importancia (puntuaciones de 1 o 2) y el $21 \%$ piensa que sí se le da suficiente importancia (puntuaciones de 4 o 5). En la importancia dada en la residencia las opiniones están más divididas, dado que el $30 \%$ se muestra indiferente, mientras que el $28 \%$ piensa que la importancia dada es insuficiente y el $31 \%$ considera que es adecuada.

\section{Experiencia en el aprendizaje de la electrocardiografía}

Los cursos presenciales han sido utilizados (puntuaciones de 4 o 5 ) por el $57 \%$ de los encuestados, mientras que no han sido utilizados por el $20 \%$ de los encuestados; el resto (16\%) se muestra indiferente. Con respecto a las charlas magistrales, el $20 \%$ se muestra indiferente, mientras que el $44 \%$ lo ha utilizado y el $29 \%$, no. Los casos clínicos que presentan sólo el trazado del ECG han sido utilizados por el $51 \%$ y no han sido utilizados por el $24 \%$, mientras que el $17 \%$ es indiferente. Los casos clínicos que presentan, además del registro electrocardiográfico, todos los datos del paciente han sido utilizados por el $54 \%$ de los encuestados, no han sido utilizados por el $22 \%$ y se muestran indiferentes el 15\%. El 65\% de los médicos ha utilizado el autoaprendizaje con un libro, método que no ha sido utilizado por el $10 \%$, mientras que permanecen indiferentes al respecto el $18 \%$. El autoaprendizaje con un CD ha sido utilizado por el $40 \%$ de los casos, 
Tabla I. Grado de conocimiento sobre los métodos de e-docencia en la enseñanza de la electrocardiografía (\%).

\begin{tabular}{lccc}
\hline & Sin experiencia (1 0 2) & Indiferente (3) & Experiencia (4 0 5) \\
\hline Proyectos de trabajo & 43 & 19 & 31 \\
\hline Visitas a sitios web & 23 & 24 & 46 \\
\hline Estudios de casos & 32 & 25 & 35 \\
\hline Visionado de documentos & 28 & 21 & 40 \\
\hline Estudio de ejemplos & 30 & 20 & 41 \\
\hline Edublogs & 65 & 12 & 14 \\
\hline Presentaciones de alumnos & 53 & 16 & 7 \\
\hline Caza del tesoro & 70 & 12 & 10 \\
\hline Sistemas 'wiki' & 75 & 8 & 22 \\
\hline Dispositivos portátiles & 53 & 18 & 72 \\
\hline
\end{tabular}

mientras que el $34 \%$ de los casos no lo ha utilizado y el $19 \%$ es indiferente. El autoaprendizaje individual a través de Internet ha sido utilizado por el $22 \%$ de los casos, mientras que el $40 \%$ no ha utilizado este procedimiento y el $19 \%$ se muestra indiferente. Las tutorías a través de Internet han sido utilizadas por el $26 \%$ de los casos, mientras que el $62 \%$ no conoce el método y el $14 \%$ se muestra indiferente.

La mayoría de los encuestados ha utilizado métodos de aprendizaje individual. El 44\% de los casos tiene experiencia (puntuaciones de 4 o 5 ) en el uso de métodos individuales, mientras que el $29 \%$ de los encuestados no ha utilizado el método (puntuaciones de 1 o 2) y el $18 \%$ se muestra indiferente. Con respecto a los métodos basados en actividades de colaboración, el $28 \%$ lo ha utilizado, mientras que el $47 \%$ no lo ha utilizado y el $18 \%$ se muestra indiferente.

\section{Grado de conocimiento de las actividades de e-docencia (Tabla I)}

El 38\% de los encuestados reconoce tener experiencia en el uso de los métodos de e-docencia, de forma que un $15 \%$ ha realizado más de 10 actividades de e-docencia, mientras que el resto reconoce haber realizado un número inferior de estas actividades, con una amplia distribución de frecuencias. Con respecto a los proyectos de trabajo realizados a través de Internet, el 31\% los conoce (puntuaciones de
4 o 5), mientras que el $43 \%$ reconoce no conocerlos (puntuaciones de 1 o 2 ) y el $19 \%$ se muestra indiferente. Por el contrario, las visitas a sitios web son utilizadas por el $46 \%$ de los casos, siendo indiferentes el $24 \%$ y reconociendo no haberlos utilizado el 23\%. El 35\% ha utilizado el estudio de casos, mientras que el $32 \%$ de los encuestados no lo ha utilizado y el 25\% de los encuestados se muestra indiferente.

El análisis mediante la lectura y visionado de documentos ha sido utilizado por el $40 \%$ de los casos, mientras que el $28 \%$ de los encuestados no lo utiliza y el $21 \%$ se muestra indiferente. El estudio de ejemplos ha sido utilizado por el $41 \%$ de los encuestados, mostrándose indiferente el $20 \%$ y reconociendo no haberlo utilizado el $30 \%$.

Los edublogs no son conocidos por el $65 \%$ de los médicos encuestados, mientras que sí los conocen el $14 \%$ y el $12 \%$ se muestra indiferente. De igual forma, el 53\% de los encuestados no ha utilizado las presentaciones de los alumnos, mientras que el 16\% es indiferente y el $22 \%$ sí lo ha utilizado. Los círculos de aprendizaje no han sido utilizados por el $59 \%$, mientras que el $19 \%$ se muestra indiferente y el $12 \%$ sí reconoce haberlo utilizado.

La caza del tesoro ha sido utilizada sólo por el 9\% de los casos, mientras que el $70 \%$ no lo ha utilizado y el $12 \%$ se muestra indiferente. Algo parecido ocurre con los sistemas 'wiki', que han sido utilizados sólo por el $10 \%$, mientras que no lo ha utilizado el $75 \%$ y se muestra indiferente el $8 \%$. El 22\% tiene experiencia en el uso de aplicaciones en dispositivos portátiles, mientras que el 53\% no lo ha utilizado y el $18 \%$ se muestra indiferente. En resumen, las visitas a sitios web y los estudios de ejemplos a través de Internet son las TIC más frecuentemente utilizadas por los médicos encuestados, y aún en un porcentaje inferior al $50 \%$.

La edad media de quienes utilizan Internet $(46,4$ años) es algo menor que la edad de quienes no lo utilizan (55,0 años), aunque este último grupo es muy pequeño. Lo mismo ocurre con respecto al uso del correo electrónico. No hay diferencias de edad entre los que han considerado la charla magistral como muy útil o poco útil, al igual que ocurre con el caso clínico, el autoaprendizaje con un libro, el autoaprendizaje con CD, el autoaprendizaje en Internet y las tutorías por Internet.

\section{Opinión sobre la posible utilidad docente del uso de las e-actividades en la enseñanza de la electrocardiografía (Tabla II)}

Los proyectos de trabajo son considerados como potencialmente útiles por el $53 \%$ de los casos, mien- 
tras que el $15 \%$ no los considera útiles y el $22 \%$ se muestra indiferente.

El 57\% de los médicos encuestados considera que la visita a sitios web puede ser útil, mientras que el $13 \%$ piensa lo contrario y el $19 \%$ se muestra indiferente. El estudio de casos es considerado útil por el $64 \%$, mientras que no lo considera útil el $12 \%$ y se muestra indiferente el 13\%. El 61\% de los encuestados piensa que el análisis, lectura y visionado de documentos puede ser útil, mientras que el $12 \%$ no piensa en su posible utilidad y el $17 \%$ se muestra indiferente. Los estudios de ejemplos son considerados potencialmente útiles por el $63 \%$ de los casos, mientras que el $12 \%$ se muestra indiferente y el $13 \%$ no considera que este método pueda ser útil. La opinión con respecto a los edublogs está más dividida, de forma que el $22 \%$ considera que pueden ser útiles, el $24 \%$ se muestra indiferente y el $31 \%$ considera que no serían útiles.

El $46 \%$ considera como potencialmente útiles las presentaciones de alumnos, el $20 \%$ se muestra indiferente y el $22 \%$ considera que este método no sería útil. Los círculos de aprendizaje son considerados útiles por el $45 \%$ de los médicos encuestados, mientras que el $18 \%$ se muestra indiferente y el $25 \%$ considera que este método no es eficaz en la enseñanza de la electrocardiografía. La caza del tesoro es considerada como potencialmente útil por el $25 \%$ de los encuestados, mientras que el $22 \%$ no lo considera útil y el $27 \%$ se muestra indiferente. El $23 \%$ de los médicos encuestados considera que los sistemas 'wiki' pueden ser útiles en la enseñanza de la electrocardiografía, el $41 \%$ no los considera útiles y el $22 \%$ se muestra indiferente. Las aplicaciones en los dispositivos portátiles son consideradas como potencialmente útiles por el $50 \%$ de los médicos encuestados, mientras que el $18 \%$ se muestra indiferente y el $20 \%$ cree que no son potencialmente útiles. En resumen, según los médicos encuestados, los estudios de ejemplos y los estudios de casos son los métodos potencialmente más útiles, mientras que los edublogs y los sistemas 'wiki' resultan los menos útiles para la enseñanza de la electrocardiografía.

\section{Diferencias entre distintos subgrupos de médicos}

Los médicos que trabajan en medio urbano utilizan Internet con mayor frecuencia que aquellos que lo hacen en un medio rural (95,3\% frente a $82,3 \%$; $p=$ 0,0058 ); también se han observado diferencias significativas en cuanto al uso de videoproyectores o cañones de proyección, CD interactivo y tableta digital (Fig. 1).
Figura 1. Comparación entre médicos que trabajan en medio urbano y rural en cuanto al porcentaje de uso de determinadas TIC. ${ }^{a} p<0,01$.

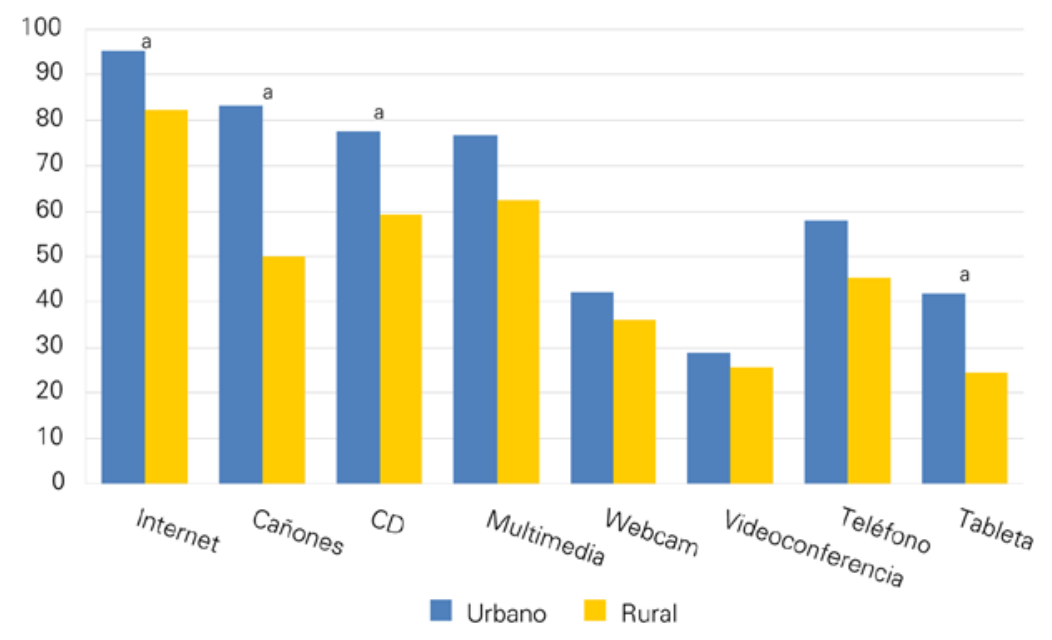

Tabla II. Opinión sobre la posible utilidad de los métodos de e-docencia en la enseñanza de la electrocardiografía (\%).

\begin{tabular}{lccc}
\hline & $\begin{array}{c}\text { Potencialmente } \\
\text { no útil (1 0 2) }\end{array}$ & $\begin{array}{c}\text { Indiferente } \\
(3)\end{array}$ & $\begin{array}{c}\text { Potencialmente } \\
\text { útil (4 0 5) }\end{array}$ \\
\hline Proyectos de trabajo & 15 & 22 & 53 \\
\hline Visitas a sitios web & 13 & 19 & 57 \\
\hline Estudios de casos & 12 & 13 & 64 \\
\hline Visionado de documentos & 12 & 13 & 61 \\
\hline Estudio de ejemplos & 13 & 12 & 63 \\
\hline Edublogs & 31 & 24 & 46 \\
\hline Presentaciones de alumnos & 22 & 20 & 25 \\
\hline Caza del tesoro & 27 & 22 & 23 \\
\hline Sistemas 'wiki' & 41 & 22 & 50 \\
\hline Dispositivos portátiles & 20 & 18 & \\
\hline
\end{tabular}

No se han observado diferencias con respecto al uso de Internet entre los médicos con una edad $\leq$ 40 años y aquellos con una edad > 40 años $(94,8 \%$ frente a $90,0 \% ; p=0,4169)$, aunque sí en cuanto al uso de webcam, curiosamente a favor de los de edad 
Figura 2. Comparación entre médicos con edad $\leq 40$ años y con edad superior a ésta en cuanto al porcentaje de uso de determinadas TIC. a $p<0,05$.

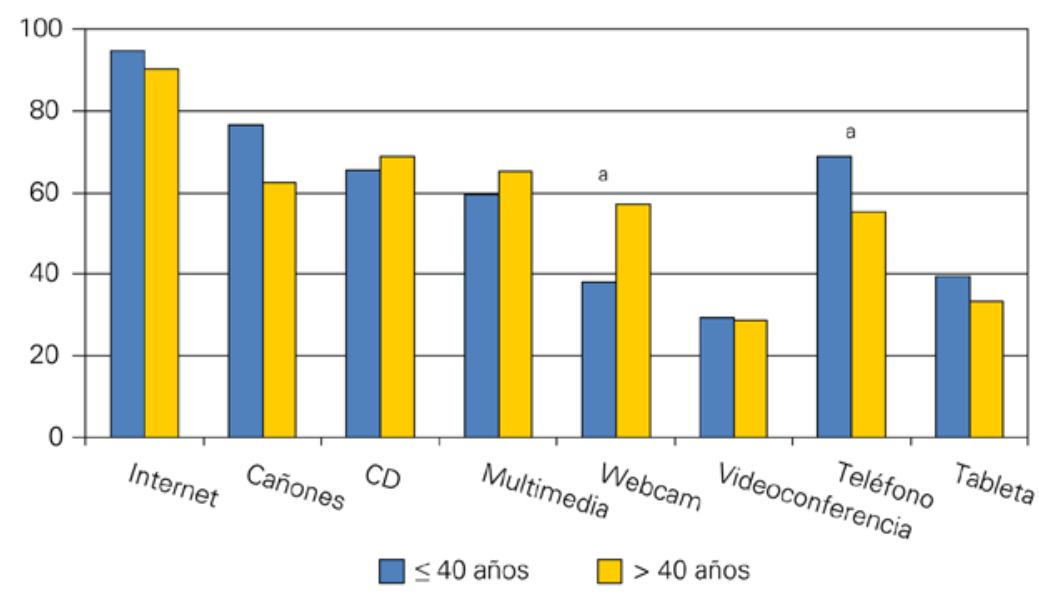

Figura 3. Comparación entre mujeres y hombres en cuanto al porcentaje de uso de determinadas TIC. a $p<0,05$.

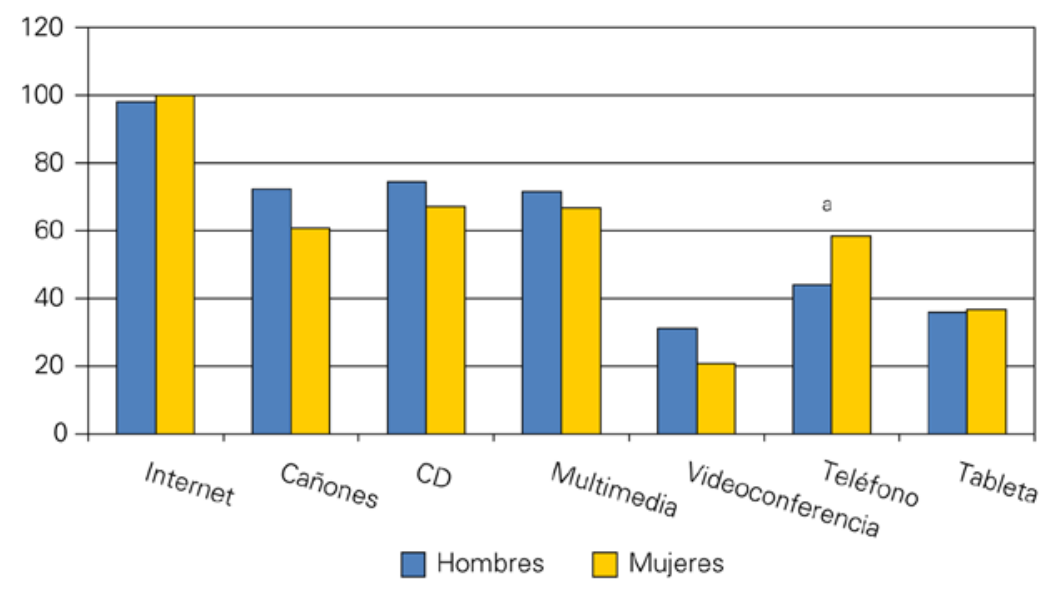

$>40$ años, y del teléfono inteligente, cuyo uso es más frecuente en los de edad $\leq 40$ años (Fig. 2).

Al comparar los hábitos de uso de TIC entre hombres y mujeres tampoco se han observado diferencias en cuento al uso de Internet ni en el hábito de uso de ninguna de las TIC consideradas (Fig. 3). El aprendizaje de la electrocardiografía es igualmente importante para todos lo médicos encuestados, dado que no hay diferencias en la edad media de los médicos que han aprendido electrocardio-
Tabla III. Estudio de las diferencias observadas entre opinión-conocimientos que muestran los encuestados sobre cada una de las metodologías docentes consideradas (puntuación media de las cuestiones).

\begin{tabular}{lc}
\hline Proyectos de trabajo & 0,8370787 \\
\hline Visitas a sitios web & 0,3050847 \\
\hline Estudios de casos & 0,7919075 \\
\hline Análisis, lectura y visionado & 0,6034483 \\
\hline Estudio de ejemplos & 0,5847953 \\
\hline Edublogs & 0,9820359 \\
\hline Presentaciones de alumnos & 0,9885057 \\
\hline Círculos de aprendizaje & 1,1556886 \\
\hline Caza del tesoro & 1,0887574 \\
\hline Sistemas 'wiki' & 0,9352941 \\
\hline Aplicaciones en dispositivos & 1,1371429 \\
\hline
\end{tabular}

grafía y los que no lo han hecho (46,2 frente a 46,9 años, respectivamente). El 30,5\% de las mujeres ha estudiado electrocardiografía, mientras que lo ha hecho el 35,0\% de los hombres. El 77,8\% de los médicos residentes ha aprendido electrocardiografía, mientras que el $70,0 \%$ de los médicos no residentes lo ha hecho. El medio laboral en el que se trabaja no parece influir en el hecho de aprender electrocardiografía, dado que lo han intentado el 69,6\% de los médicos que trabajan en medio urbano y el 70,7\% de quienes lo hacen en un medio rural. El 74,2\% de los médicos de atención primaria ha estudiado electrocardiografía, mientras que el 70,7\% de los de otras especialidades (excepto cardiología, en la que todos lo han hecho) han estudiado esta técnica diagnóstica $(p=0,1103)$.

El 75,0\% de los médicos que trabajan en el Área Sanitaria de Talavera de la Reina han aprendido electrocardiografía, frente al $69,6 \%$ de los que trabajan en el Área Sanitaria de Toledo $(p=0,6494)$.

$\mathrm{Al}$ analizar las diferencias entre la puntuación obtenida en las cuestiones que evalúan el conocimiento y la alcanzada en aquellas otras que valoran la opinión sobre las distintas TIC, la diferencia ha sido siempre positiva (Tabla III), lo que indica que los sujetos tienden a valorar más aquello que conocen. En consonancia con esto se ha observado una correlación significativa positiva entre las puntuaciones obtenidas para cada una de las TIC en las 
Tabla IV. Correlación (coeficiente de Pearson) entre el grado de conocimiento y de opinión de los médicos encuestados sobre cada una de las metodologías docentes consideradas ( $p<0,05$ en todas las indicadas).

\begin{tabular}{|c|c|c|c|c|c|c|c|c|c|c|c|}
\hline & \multicolumn{11}{|c|}{ Conocimiento } \\
\hline & P. trabajo & Web & Casos & Documentos & Ejemplos & Blogs & Alumnos & Círculos & Caza & Wiki & Apps \\
\hline P. trabajo & 0,23020 & & & & & & & & & & \\
\hline Web & & 0,31245 & & & & & & & & & \\
\hline Casos & & & 0,25390 & & & & & & & & \\
\hline Documentos & & & & 0,24819 & & & & & & & \\
\hline Ejemplos & & & & & 0,29742 & & & & & & \\
\hline Blogs & & & & & & 0,32489 & & & & & \\
\hline Alumnos & & & & & & & 0,37082 & & & & \\
\hline Círculos & & & & & & & & 0,31829 & & & \\
\hline Caza & & & & & & & & & 0,3491 & & \\
\hline Wiki & & & & & & & & & & 0,3220 & \\
\hline Apps & & & & & & & & & & & 0,3425 \\
\hline
\end{tabular}

cuestiones que evalúan conocimiento y opinión (Tabla IV).

Los médicos especialistas en cardiología mostraron mayor interés en el conocimiento de la electrocardiografía, aunque no se observaron diferencias con respecto a la técnica utilizada para su aprendizaje con respecto al resto de los especialistas ni en el grado de conocimiento de las técnicas de e-docencia.

\section{Discusión}

En este trabajo se ha documentado que los médicos de la provincia de Toledo encuestados tienen interés por el aprendizaje de la electrocardiografía, utilizan el ordenador en su vida diaria, usan primordialmente métodos de estudio individuales y tienen un escaso conocimiento de las TIC utilizadas en la docencia de la medicina. Además, los médicos que trabajan en el área urbana hacen un mayor uso de Internet y tienen más conocimiento de las TIC dedicadas a la docencia, mientras que no se han observado diferencias significativas entre sexos y sólo pequeñas diferencias con la edad.

La mayoría de los médicos encuestados tiene experiencia en el aprendizaje de la electrocardiogra- fía, aunque ha utilizado métodos de aprendizaje que podríamos considerar 'tradicionales' (cursos y charlas presenciales), lo cual es lógico dado que han sido las técnicas utilizadas con mayor frecuencia, a pesar de que opinan que no se da suficiente importancia al aprendizaje de la electrocardiografía, especialmente en la universidad. En la residencia sí se da más importancia al aprendizaje de la electrocardiografía, lo cual tiene sentido sobre todo en las especialidades médicas, ya que un adecuado conocimiento de la electrocardiografía es necesario para la asistencia.

La mayoría de los médicos encuestados tiene experiencia en el uso de actividades individuales para el aprendizaje de la electrocardiografía, mientras que la experiencia en el uso de las actividades de colaboración es bastante menor. Esto puede explicarse porque la enseñanza tradicional de la medicina se ha realizado más con procedimientos de aprendizaje individual que colectivo, lo cual está cambiando en los últimos años. Las TIC pueden contribuir a desarrollar el hábito de trabajo en grupo porque determinadas estrategias metodológicas promueven el trabajo en grupo y colaborativo [1]. El cambio que las TIC producen en el alumno (más activo) y el profesor (más facilitador e informador) será muy positivo en la adaptación de los médicos 
al uso de la información y a un trabajo de mayor colaboración, como de hecho se ha producido en la práctica de la medicina [26].

De los métodos de autoaprendizaje, el libro es el más utilizado por los encuestados, con un uso bastante menos frecuente del CD y de Internet. Estos resultados parecen lógicos, dada la marcada tradición del uso del libro en el aprendizaje como elemento casi exclusivo, complementado en las últimas décadas con el uso del CD y de Internet, más como elementos para la presentación de casos que como sustitutos del libro. Resultados similares han sido comunicados por otros autores en estudiantes de medicina [27]. Estos métodos de aprendizaje están cambiando en los últimos años: se está imponiendo en las facultades de medicina el aprendizaje basado en la resolución de problemas, lo cual puede ser un excelente complemento a los métodos de docencia tradicionales en una formación científicotécnica como la que es necesaria en medicina [28]. Por el contrario, existe poca experiencia en la utilización de proyectos colaborativos en la enseñanza de la medicina [29], aunque es probable que esta experiencia se incremente en nuestro país con la modificación de la enseñanza (Plan de Bolonia), que conlleva mayor actividad de colaboración en la enseñanza [1].

El papel e impacto de las TIC en la docencia se ha estudiado desde hace tiempo. Se han realizado estudios en la Universidad de Edimburgo, donde se recogen datos acerca de las experiencias, conocimientos y actitudes ante las TIC de los nuevos estudiantes desde 1990 [30]. Posteriormente, el informe SEUSSIS (2003) evaluó el uso de las TIC en siete universidades europeas (Reino Unido, Finlandia, Noruega, Países Bajos, Italia, Francia y España), recogiendo datos de los años 2001-2002 [31]. Este tipo de estudios se han realizado principalmente en alumnos de medicina $[6,8,9,32]$ y profesionales de la salud [7] y han mostrado una situación muy similar a la descrita en nuestro trabajo, aunque con las diferencias propias del tiempo y de la progresiva adopción de las TIC.

A la hora de preguntar sobre la potencial utilidad de los métodos presentados para el aprendizaje de la electrocardiografía, los médicos consideran como más útiles a los estudios de casos y ejemplos las visitas a páginas web y el análisis y visionado de documentos, mientras que no consideran que los blogs educativos puedan ser de utilidad. Esto se debe probablemente al desconocimiento que muestran sobre los edublogs, dado que pueden ser una herramienta más para ver y analizar documentos vinculados a los mismos. Es de esperar que un me- jor conocimiento de las TIC, lo que indudablemente se producirá de forma progresiva, les hará valorar mejor lo que estos distintos métodos pueden aportar en cada caso. El hecho de que permitan un trabajo personalizado, con análisis de documentos y de abundante información [33], al igual que ocurre con los portafolios electrónicos [34], puede adaptarse a las características y hábitos de trabajo de los médicos mejor que otros métodos.

Los médicos sí son conscientes de la potencial utilidad de los teléfonos inteligentes en la docencia, pues aunque su uso no está todavía demasiado extendido, la mayoría de los encuestados considera que pueden ser de utilidad para el aprendizaje de la electrocardiografía. Es bien conocido que el teléfono móvil se ha convertido en el medio de comunicación más extendido, que forma parte de un mercado en continua expansión y que, además, está desarrollando continuamente nuevas prestaciones, muchas de las cuales tienen un gran potencial para la formación, lo que ha dado lugar a un nuevo paradigma educativo conocido como mobile learning o aprendizaje móvil [35].

Aunque no se ha preguntado expresamente sobre las redes sociales como medios de aprendizaje, lo cierto es que podemos deducir cuál sería su utilidad como medio de soporte de los distintos métodos de aprendizaje considerados (casos, visión de documentos, etc.), dada su similitud. La continua expansión de estas redes, y su diversificación en búsqueda de distintos perfiles de usuarios, está abocando a que se utilicen de manera creciente en el proceso de enseñanza-aprendizaje [36]. Por ello es de esperar que en un futuro inmediato ocupen un papel importante, aunque deben superarse las incertidumbres, limitaciones y riesgos que todavía presentan las redes sociales. De esta forma, las denominadas 'escuelas 2.0' y el 'tecnoconocimiento' aportarán un impulso importante a la docencia, contribuyendo a un nuevo paradigma en las aulas $[37,38]$.

En nuestra muestra se observa que los médicos que viven en medio urbano utilizan Internet con una frecuencia significativamente mayor que aquellos que viven en zonas rurales, aunque en ambos grupos la frecuencia de uso es elevada. Es interesante subrayar que, por el contrario, los médicos menores de 40 años no utilizan Internet con mayor frecuencia, aunque sí hacen mayor uso de los teléfonos inteligentes, probablemente debido al hecho de que este grupo etario está más expuesto a esta técnica. Esto puede deberse a que desde hace años se viene utilizando Internet como medio de consulta y de información en medicina, por lo que no se observan diferencias entre los médicos según sus 
edades en cuanto a la frecuencia de uso de este método. Por el contrario, los teléfonos inteligentes son más recientes y, lógicamente, están más difundidos entre los médicos más jóvenes, dado su mayor uso de las redes sociales, a los que estos sistemas están muy vinculados. A pesar de ello, sorprende el escaso conocimiento de los médicos sobre el uso de aplicaciones en dispositivos portátiles como herramientas de aprendizaje de la electrocardiografía.

Este estudio tiene algunas limitaciones que conviene considerar. En primer lugar, existe sesgo en la selección de la muestra porque la encuesta se ha remitido sólo a médicos que utilizan el correo electrónico en sus comunicaciones. No obstante, la mayoría de los médicos colegiados utilizan el correo electrónico, por lo que consideramos que puede ser razonable asumir que este sesgo no es tan marcado como para invalidar nuestros resultados. De igual forma, la muestra no es aleatoria, a pesar de lo cual consideramos que el número de encuestas recibido parece representativo y puede ser razonable extrapolar estos datos a la muestra, asumiendo que los resultados globales pueden ser incluso peores en la muestra total en lo que al conocimiento de las TIC se refiere porque es probable que hayan respondido con más frecuencia los que más interés tienen en las TIC.

En conclusión, los datos de este estudio indican una adopción algo desigual de las TIC, con pequeñas diferencias según las características consideradas (sexo, edad, medio laboral), pero muestran una visión positiva de la mayoría sobre el potencial de las TIC en la enseñanza de la medicina.

\section{Bibliografía}

1. Prendes-Espinosa MP. Internet aplicado a la educación: estrategias didácticas y metodológicas. In Cabero-Almenara J, coord. Nuevas tecnologías aplicadas a la educación. Madrid McGraw-Hill; 2007. p. 205-22.

2. The future of medicine. Squeezing out the doctor. The role of physicians at the centre of health care is under pressure. The Economist, 2 de junio de 2012.

3. Landolina M, Perego GB, Lunati M, Curnis A, Guenzati G, Vicentini A, et al. Remote monitoring reduces healthcare use and improves quality of care in heart failure patients with implantable defibrillators: the evolution of management strategies of heart failure patients with implantable defibrillators (EVOLVO) study. Circulation 2012; 125: 2985-92.

4. Chang G, Cook D, Maguire T, Skakun E, Yakimets WW, Warnock GL. Problem-based learning: its role in undergraduate surgical education. Can J Surg 1995; 38: 13-21.

5. Nandi PL, Chan JN, Chan CP, Chan P, Chan LP. Undergraduate medical education: comparison of problem-based learning and conventional teaching. Hong Kong Med J 2000; 6: 301-6.

6. Dorup J. Experience and attitudes towards information technology among first-year medical students in Denmark: longitudinal questionnaire survey. J Med Internet Res 2004; 6: 10.

7. Jadad AR, Dphil SC, Cocking LB, Lynda BA, Whelan T, Browman G. Internet use among physicians, nurse, and their patients. JAMA 2001; 286: 1451-2.
8. Gouveia-Oliveira A, Rodrigues T, De Melo FG. Computer education: attitudes and opinions of first-year medical students. Med Educ 1994; 28: 501-7.

9. Nurjahan MI, Lim TA, Foong A, Yeonf SW, Ware J. Computer literacy in medical students. Med Educ 2000; 34: 966.

10. Bravo-Toledo R. Contra la resistencia, innovación. Cibermedicina 2011; 1: 3.

11. Hoyle RJ, Walker KJ, Thomson G, Bailey M. Accuracy of electrocardiogram interpretation improves with emergency medicine training. Emerg Med Australas 2007; 19: 143-50.

12. Margolis S, Reed R. EKG analysis skills of family practice residents in the United Arab Emirates: a comparison with US data. Fam Med 2001; 33: 447-52.

13. Boltri JM, Hash RB, Vogel RL. Are family practice residents able to interpret electrocardiograms? Adv Health Sci Educ 2003; 8: 149-53.

14. Larson DM, Menssen KM, Sharkey SW, Duval S, Schwartz RS, Harris J, et al. 'False-positive' cardiac catheterization laboratory activation among patients with suspected ST-segment elevation myocardial infarction. JAMA 2007; 298: 2754-60.

15. Berger JS, Eisen L, Nozad V, D’Angelo J, Calderon Y, Brown DL, et al. Competency in electrocardiogram interpretation among internal medicine and emergency medicine residents. Am J Med 2005; 118: 873-80.

16. Jensen MS, Thomsen JL, Jensen SE, Lauritzen T, Engberg M. Electrocardiogram interpretation in general practice. Fam Pract 2005; 22: 109-13.

17. Schultz, KK, Brackbill ML. Teaching electrocardiogram basics using dance and movement. Am J Pharm Educ 2009; 73: 70.

18. Owen SG. Electrocardiography. London: English Universities Press; 1966.

19. Cuddy TE, Kaufman BJ. A comparison between a programmed text and a lecture course in electrocardiography. CMAJ 1970; 102: 971.

20. Calderón-Segura JA, Castaño-Castrillón JJ, Castaño-Valencia O, Pinilla-Cañón M, Ruano-Vargas MA, Garzón-Rodríguez N, et al. Evaluación del conocimiento del patrón electrocardiográfico en estudiantes de medicina de la Universidad de Manizales de octavo a décimo semestre. Año 2008. Arch Med (Manizales) 2009; 9: 13-24.

21. Nilsson M, Bolinder G, Held C, Johansson BL, Fors U, Östergren J. Evaluation of a web-based ECG-interpretation programme for undergraduate medical students. BMC Med Educ 2008; 8: 25 .

22. Rubinstein J, Dhoble A, Ferenchick G. Puzzle based teaching versus traditional instruction in electrocardiogram interpretation for medical students -a pilot study. BMC Med Educ 2009; 9: 4.

23. Alarco JJ, Álvarez-Andrade EV. Google Docs. Una alternativa de encuesta online. Educ Med 2012; 1: 9-10.

24. Lara-Guijarro E, Ballesteros-Velázquez B. Métodos de investigación en educación social. Madrid: UNED; 2007.

25. Cabero-Almenara J, Román-Graván P, coords. E-actividades. Un referente básico para la formación en Internet. Sevilla: Eduforma; 2005.

26. Cabero-Almenara J, Gisbert-Cervera M. El papel del profesor y el alumno en los nuevos entornos tecnológicos de formación. In Cabero-Almenara J, coord. Nuevas tecnologías aplicadas a la educación. Madrid: McGraw-Hill: 2007. p. 261-77.

27. Gil MC, Arnedo M, Ribate MP, Puisac B, Lanuza J, Sáenz MA, et al. Uso de las tecnologías de la información y comunicación (TIC) en las licenciaturas de bioquímica y medicina de la Universidad de Zaragoza. IX Encuentro Virtual Educa Zaragoza; 2008.

28. González-López E, García-Lázaro I, Blanco-Alfonso A, Otero-Puime A. Aprendizaje basado en la resolución de problemas: una experiencia práctica. Educ Med 2010; 13: 15-24.

29. Parra P, Matus O, Ortiz L, Torres G, Ibacaches A, Ramírez L, et al. Enseñanza y evaluación del profesionalismo médico a través de proyectos colaborativos. Rev Educ Cienc Salud 2010; 7: 29-34.

30. Asgari-Jirhandeh N, Haywood J. Computer awareness among medical students: a survey. Med Educ 1997; 31: 225-9.

31. Haywood J, Haywood D. ICT Skills of Students in European 
Universities: the SEUSISS Project Final Report. Edinburgh: Scottish Centre for Research into Online Learning \& Assessment; 2003.

32. Valcke M, De Wever B. Information and communication technologies in higher education: evidence-based practices in medical education. Med Teach 2006; 28: 40-8.

33. Roig-Vila I. Internet aplicado a la educación; webquest, wiki y weblog. In Cabero-Almenara J, coord. Nuevas tecnologías aplicadas a la educación. Madrid: McGraw-Hill; 2007. p. 223-43.

34. Cacheiro-González ML. El porfolio electrónico como estrategia de apropiación de competencias. In Gallego-Gil D, Alonso-García C, Cacheiro-González ML, coords. Educación, sociedad y tecnología. Madrid: Ramón Areces/UNED; 2011. p. 291-306.
35. Brazuelo-Grund F. Nuevos recursos móviles. In Gallego-Gil D, Alonso-García C, Cacheiro-González ML, coords. Educación sociedad y tecnología. Madrid: Ramón Areces/UNED; 2011. p. 337-66.

36. Fumero A. Somos redes; somos profesionales. Un breve recorrido por la realidad de las redes sociales profesionales. Cibermedicina 2011; 1: 4-7.

37. Atrio-Cerezo S. Redes sociales. In Gallego-Gil D, AlonsoGarcía C, Cacheiro-González ML, coords. Educación, sociedad y tecnología. Madrid: Ramón Areces/UNED; 2011. p. 307-36.

38. Alonso-García C, Gallego DJ. Tecnocimiento: conocimiento y tecnología. In Gallego-Gil D, Alonso-García C, CacheiroGonzález ML, coords. Educación, sociedad y tecnología. Madrid: Ramón Areces/UNED; 2011. p. 1-37. 


\section{CUESTIONARIO}

ENCUESTA SOBRE EL CONOCIMIENTO Y USO DE LAS TECNOLOGÍAS DE LA INFORMACIÓN Y DE LA COMUNICACIÓN EN LA ENSEÑANZA DE LA ELECTROCARDIOGRAFÍA.

Dr. Luis Rodríguez Padial

Se pretende analizar el grado de conocimiento de las TIC (tecnologías de la información y comunicación) y, específicamente, su uso en la enseñanza y aprendizaje de la electrocardiografía. Por favor, conteste el cuestionario, lo que le llevará cinco minutos, y remítalo una vez finalizado dando a "enviar".

En las preguntas de elección múltiple, el 0 significa siempre que no conoce el método indicado. Los otros valores pueden indicar, según la serie de preguntas, el grado de conocimiento -muy conocidos (5) y nada conocidos (1)-, que está de totalmente de acuerdo (5) o totalmente en desacuerdo (1) con la afirmación o que cree que son muy útiles (5) o nada útiles (1).

Es importante señalar que no hay respuestas correctas o incorrectas.

Por favor, conteste el cuestionario una sola vez.

Muchas gracias por su colaboración.

Luis Rodríguez Padial

Carddiólogo, Toledo

\section{DATOS SOCIO-ECONÓMICOS}

\section{Edad}

\section{Género}

Femenino

Г Masculino

\section{Nacionalidad}

\section{Profesión \\ $\lceil$ Medicina \\ Г Enfermería}
Medio laboral
ए Rural
ГUrbano

\section{Provincia en la que trabaja}

Si trabaja en varias provincias, considere solo en la que tiene su trabajo principal

$\Gamma$ Toledo 
Г Otra

De trabajar en Toledo, indique el área sanitaria en la que trabaja

$\Gamma$ Toledo

Г Talavera de la Reina

\section{Médico Residente}

$\Gamma \mathrm{Si}$

$\Gamma$ No

\section{Especialidad}

$\Gamma$ Atención primaria

Г Cardiología

$\Gamma$ Otra

\section{Año de Licenciatura}

\section{Aprendizaje de ECG}

Ha realizado el aprendizaje de la electrocardiografía mediante algún método didáctico

$\Gamma \mathrm{Si}$

$\Gamma$ No

\section{Nivel de formación en ECG}

Indicar cuál cree que es su nivel de formación en electrocardiografía

$\Gamma$ Ninguna

Г Básica

$\Gamma$ Intermedia

$\Gamma$ Avanzada

\section{EXPERIENCIA DE USO DE LAS TIC EN LABORES PERSONALES}

Indicar si utiliza habitualmente las TIC señaladas en la vida diaria para sus actividades personales

\section{Ordenadores}

No los usa

Los usa 
Cañones (videoproyectores)

No los usa

- Los usa

\section{Interactivos}

No los usa

- Los usa

\section{Equipos multimedia}

C No los usa

Los usa

Internet

No lo usa

Lo usa

\section{Correo electrónico}

C No lo usa

C Lo usa

\section{Webcam}

- No la usa

- La usa

\section{Equipo de videoconferencia \\ . No lo usa \\ C Lo usa}

Tableta electrónica (iPad y similares)

No lo usa

Lo usa

Teléfono inteligente (iPhone y similares)

No lo usa

Lo usa 


\section{IMPORTANCIA DE LA ENSEÑANZA-APRENDIZAJE DE LA ELECTROCARDIOGRAFÍA}

Se pretende conocer su opinión sobre la importancia que se da en la actualidad a la enseñanzaaprendizaje de la electrocardiografía en la Universidad y en la Residencia

(1: totalmente en desacuerdo; 2: en desacuerdo; 3; ni de acuerdo ni en desacuerdo; 4: de acuerdo; 5: totalmente de acuerdo)

Considera que se da suficiente importancia a la enseñanza-aprendizaje de la electrocardiografía en la Universidad

$$
\begin{array}{lllll}
1 & 2 & 3 & 4 & 5
\end{array}
$$

No acuerdo $\bigcirc 0 \% \bigcirc \bigcirc$ Acuerdo

Piensa que se da suficiente importancia a la enseñanza-aprendizaje de la electrocadiografía en el período de Residencia

$\begin{array}{lllll}1 & 2 & 3 & 4 & 5\end{array}$

No acuerdo $000 \% 0$ Acuerdo

\section{EXPERIENCIA CON EL APRENDIZAJE DE LA ELECTROCARDIOGRAFÍA}

Se pretende conocer su experiencia sobre el uso de determinadas metolologías de aprendizaje, algunas relacionadas con las TIC, en la enseñanza-aprendizaje de la electrocardiografía

(1: totalmente desconocido; 2: poco conocido; 3; indeciso; 4: algo conocido; 5 : totalmente conocido)

\section{Cursos presenciales}

\begin{tabular}{lllllll} 
& 1 & 2 & 3 & 4 & 5 & \\
\hline No conocido & $\bigcirc$ & $\bigcirc$ & $\bigcirc$ & $\bigcirc$ & 0 & Conocido
\end{tabular}

Charla magistral

$\begin{array}{lllll}1 & 2 & 3 & 4 & 5\end{array}$

No conocido $0 \quad 0 \quad 0 \quad 0 \quad 0$ Conocido

Caso Clínico (sólo ECG)

$\begin{array}{lllll}1 & 2 & 3 & 4 & 5\end{array}$

No conocido $0 \quad 0 \quad 0 \quad 0 \quad 0$ Conocido

Caso Clínico (caso completo)

\begin{tabular}{lllllll} 
& 1 & 2 & 3 & 4 & 5 & \\
\hline No conocido & $\bigcirc$ & $\bigcirc$ & $\bigcirc$ & $\bigcirc$ & $\bigcirc$ & Conocido
\end{tabular}


Autoaprendizaje con Libro

\begin{tabular}{lllllll} 
& 1 & 2 & 3 & 4 & 5 & \\
\hline No conocido & $\bigcirc$ & $\bigcirc$ & $\bigcirc$ & $\bigcirc$ & $\bigcirc$ & Conocido
\end{tabular}

Autoaprendizaje con CD

$\begin{array}{lllll}1 & 2 & 3 & 4 & 5\end{array}$

No conocido $\odot \quad \odot \quad \circ \quad \circ \quad 0$ Conocido

Autoaprendizaje individual a través de Internet

\begin{tabular}{lllllll} 
& 1 & 2 & 3 & 4 & 5 & \\
\hline No conocido & $\bigcirc$ & $\bigcirc$ & $\bigcirc$ & $\bigcirc$ & $\bigcirc$ & Conocido
\end{tabular}

Tutorías por Internet

\begin{tabular}{lllllll} 
& 1 & 2 & 3 & 4 & 5 & \\
\hline No conocido & 0 & $\bigcirc$ & 0 & $\bigcirc$ & 0 & Conocido
\end{tabular}

Actividades individuales

$\begin{array}{lllll}1 & 2 & 3 & 4 & 5\end{array}$

No conocido $\odot \quad \circ \quad \circ \quad \circ \quad 0$ Conocido

Actividades de colaboración

\begin{tabular}{lllllll} 
& 1 & 2 & 3 & 4 & 5 & \\
\hline No conocido & $\bigcirc$ & $\bigcirc$ & $\bigcirc$ & $\bigcirc$ & $\bigcirc$ & Conocido
\end{tabular}

\section{GRADO DE CONOCIMIENTO DE LAS ACTIVIDADES DE E-DOCENCIA}

Se pretende conocer el grado de conocimiento que tiene sobre diversas metologías docentes que se apoyan en el uso de las TIC en la enseñanza-aprendizaje de la electrocardiografía (se preguntará después su opinión sobre las mismas)

(1: totalmente desconocido; 2 : poco conocido; 3; indeciso; 4: algo conocido; 5 : totalmente conocido)

\section{Proyectos de Trabajo}

Desarrollo a través de Internet de una activdad previamente determinada para alcanzar unos objetivos predefinidos

\begin{tabular}{lllllll} 
& 1 & 2 & 3 & 4 & 5 & \\
\hline No conocido & $\bigcirc$ & $\bigcirc$ & $\bigcirc$ & $\bigcirc$ & $\bigcirc$ & Conocido
\end{tabular}


Visitas a sitios Web

\begin{tabular}{lllllll}
1 & 2 & 3 & 4 & 5 \\
\hline No conocido & 0 & 0 & 0 & 0 & 0 & Conocido
\end{tabular}

\section{Estudios de Casos}

\begin{tabular}{lllllll} 
& 1 & 2 & 3 & 4 & 5 & \\
\hline No conocido & $\bigcirc$ & $\bigcirc$ & $\bigcirc$ & $\bigcirc$ & $\bigcirc$ & Conocido
\end{tabular}

Análisis de lectura y visionado de documentos

\begin{tabular}{lllllll} 
& 1 & 2 & 3 & 4 & 5 & \\
\hline No conocido & $\bigcirc$ & $\bigcirc$ & $\bigcirc$ & $\bigcirc$ & $\bigcirc$ & Conocido
\end{tabular}

\section{Estudio de ejemplos}

\begin{tabular}{lllllll} 
& 1 & 2 & 3 & 4 & 5 & \\
\hline No conocido & $\bigcirc$ & $\bigcirc$ & $\bigcirc$ & $\bigcirc$ & $\bigcirc$ & Conocido
\end{tabular}

\section{Edublogs}

Blogs o bitácoras que tienen como objetivo se herramientas educativas

\begin{tabular}{lllllll} 
& 1 & 2 & 3 & 4 & 5 & \\
\hline No conocido & $\bigcirc$ & $\bigcirc$ & $\bigcirc$ & $\bigcirc$ & $\bigcirc$ & Conocido
\end{tabular}

\section{Presentaciones de los alumnos}

Presentaciones en el salón virtual de clase por parte de los alumnos

$$
\begin{array}{lllll}
1 & 2 & 3 & 4 & 5
\end{array}
$$

No conocido $0 \quad 0 \quad 0 \quad 0 \quad 0$ Conocido

\section{Círculos de aprendizaje}

Ttrabajo colectivo en red en el que se aborda un problema desde distintas perspectivas

$\begin{array}{lllllll} & 1 & 2 & 3 & 4 & 5 & \\ \text { No conocido } & \bigcirc & \bigcirc & \bigcirc & \bigcirc & \bigcirc & \text { Conocido }\end{array}$

\section{Caza del tesoro}

Página web con cuestiones y direcciones electrónicas donde buscar la respuesta

\begin{tabular}{lllllll} 
& 1 & 2 & 3 & 4 & 5 & \\
\hline No conocido & $\bigcirc$ & $\bigcirc$ & $\bigcirc$ & $\bigcirc$ & $\bigcirc$ & Conocido
\end{tabular}


Sistemas que dan posibilidad a cualquier usuario autorizado de añadir o borrar contenido

\begin{tabular}{lllllll} 
& 1 & 2 & 3 & 4 & 5 & \\
\hline No conocido & $\bigcirc$ & $\bigcirc$ & $\bigcirc$ & $\bigcirc$ & $\bigcirc$ & Conocido
\end{tabular}

Aplicaciones en dispositivos portátiles

(teléfonos inteligentes, tabletas, etc.)

$\begin{array}{lllll}1 & 2 & 3 & 4 & 5\end{array}$

No conocido $0 \quad 0 \quad 0 \quad 0 \quad 0$ Conocido

Tiene experiencia previa del uso de las e-actividades en la docencia

Uso previo
No
Si

De decir si, cuantas veces

\section{OPINIÓN SOBRE LA POSIBLE UTILIDAD DOCENTE DEL USO DE E- ACTIVIDADES EN LA DOCENCIA DE LA ELECTROCARDIOGRAFÍA}

Se pretende conocer su opinión sobre la posible utilidad de diversas metologías docentes que se apoyan en las TIC en la enseñanza/aprendizaje de la electrocardiografía

(1: totalmente en desacuerdo; 2: en desacuerdo; 3; ni de acuerdo ni en desacuerdo; 4: de acuerdo; 5 : totalmente de acuerdo)

\section{Proyectos de Trabajo}

Desarrollo a través de Internet de una activdad previamente determinada para alcanzar unos objetivos predefinidos

\begin{tabular}{lllllll} 
& 1 & 2 & 3 & 4 & 5 & \\
\hline Desacuerdo & $\bigcirc$ & $\bigcirc$ & $\bigcirc$ & $\bigcirc$ & $\bigcirc$ & Acuerdo
\end{tabular}

Visitas a sitios Web

$$
\begin{array}{lllll}
1 & 2 & 3 & 4 & 5
\end{array}
$$

Desacuerdo $\bigcirc \circ \% \bigcirc$ Acuerdo

\section{Estudio de Casos}

\begin{tabular}{lllllll} 
& 1 & 2 & 3 & 4 & 5 & \\
\hline Desacuerdo & 0 & 0 & 0 & 0 & 0 & Acuerdo
\end{tabular}




\begin{tabular}{lllllll} 
& 1 & 2 & 3 & 4 & 5 & \\
\hline Desacuerdo & $\bigcirc$ & $\bigcirc$ & $\bigcirc$ & $\bigcirc$ & $\bigcirc$ & Acuerdo
\end{tabular}

Estudio de ejemplos

\begin{tabular}{lllllll} 
& 1 & 2 & 3 & 4 & 5 & \\
\hline Desacuerdo & 0 & 0 & $\bigcirc$ & $\bigcirc$ & $\bigcirc$ & Acuerdo
\end{tabular}

\section{Edublogs}

Blogs o bitácoras que tienen como objetivo se herramientas educativas

\begin{tabular}{lllllll} 
& 1 & 2 & 3 & 4 & 5 & \\
\hline Desacuerdo & $\bigcirc$ & 0 & $\bigcirc$ & $\bigcirc$ & $\bigcirc$ & Acuerdo
\end{tabular}

\section{Presentaciones de Alumnos}

Presentaciones en el salón virtual de clase por parte de los alumnos

\begin{tabular}{lllllll} 
& 1 & 2 & 3 & 4 & 5 & \\
\hline Desacuerdo & $\bigcirc$ & $\bigcirc$ & $\bigcirc$ & $\bigcirc$ & 0 & Acuerdo
\end{tabular}

\section{Círculos de Aprendizaje}

Trabajo colectivo en red en el que se aborda un problema desde distintas perspectivas

\begin{tabular}{lllllll} 
& 1 & 2 & 3 & 4 & 5 & \\
\hline Desacuerdo & $\bigcirc$ & 0 & 0 & 0 & 0 & Acuerdo
\end{tabular}

\section{Caza del tesoro}

Página web con cuestiones y direcciones electrónicas donde buscar la respuesta

\begin{tabular}{lllllll} 
& 1 & 2 & 3 & 4 & 5 & \\
\hline Desacuerdo & $\bigcirc$ & $\bigcirc$ & $\bigcirc$ & $\bigcirc$ & $\bigcirc$ & Acuerdo
\end{tabular}

\section{Sistemas Wiki}

Sistemas que dan posibilidad a cualquier usuario autorizado de añadir o borrar contenido

\begin{tabular}{lllllll} 
& 1 & 2 & 3 & 4 & 5 & \\
\hline Desacuerdo & 0 & 0 & 0 & 0 & 0 & Acuerdo
\end{tabular}

Aplicaciones en dispositivos portátiles

(teléfonos inteligentes, tabletas, etc.)

\begin{tabular}{lllllll} 
& 1 & 2 & 3 & 4 & 5 & \\
\hline Desacuerdo & 0 & 0 & $\bigcirc$ & $\bigcirc$ & 0 & Acuerdo
\end{tabular}


Indique, por favor, algún aspecto sobre el tema que le parezca interesante y que no se haya tratado

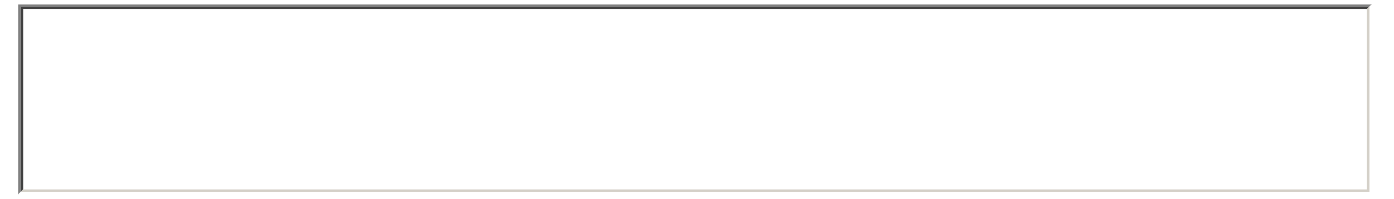

